\title{
Evaluation of Three Borate-Bioactive Glass Compositions for Antibacterial Applications
}

\author{
David Westenberg1 $^{*}$ (), Ravi Viswanathan1, Deana L. Kadyk1, Suzanne Hibbs', Jonathan Kopel², \\ Delbert Day ${ }^{3}$
}

${ }^{1}$ Biological Sciences, Missouri University of Science and Technology, Rolla, MO, USA

${ }^{2}$ Chemistry, Missouri University of Science and Technology, Rolla, MO, USA

${ }^{3}$ Material Science and Engineering, Missouri University of Science and Technology, Rolla, MO, USA

Email: ^djwesten@mst.edu

How to cite this paper: Westenberg, D. Viswanathan, R., Kadyk, D.L., Hibbs, S., Kopel, J. and Day, D. (2021) Evaluation of Three Borate-Bioactive Glass Compositions for Antibacterial Applications. Advances in Microbiology, 11, 646-656.

https://doi.org/10.4236/aim.2021.1111047

Received: October 1, 2021

Accepted: November 22, 2021

Published: November 25, 2021

Copyright $\odot 2021$ by author(s) and Scientific Research Publishing Inc. This work is licensed under the Creative Commons Attribution-NonCommercial International License (CC BY-NC 4.0). http://creativecommons.org/licenses/by-nc/4.0/

\begin{abstract}
The prevalence of cases of antibiotic resistant infections and the limited number of new antibiotics call for new strategies to prevent bacterial infections. In this study, we evaluate the effectiveness of three different compositions of bioactive glass, doped with silver as an antimicrobial agent for potential applications in preventing bacterial infections. The different glasses were formulated to dissolve and release silver at different rates enabling their use in different applications either individually or in combination to achieve a desired effect. Silver oxide $\left(\mathrm{Ag}_{2} \mathrm{O}\right)$ was incorporated into various glass formulations and antibacterial effectiveness was measured using radius of inhibition. Each silver-doped glass effectively inhibited the growth of Escherichia coli, Staphylococcus aureus and Pseudomonas aeruginosa with radius of inhibition correlating directly with the dissolution rate of the glass-the faster dissolving glass producing the larger radius of inhibition. Suspending particles of the silver containing glass in phosphate buffered saline or fetal calf serum, slowed the dissolution of each glass and extended the effectiveness of the silver containing glass over time (based on size of the radius of inhibition) compared to water. Potential applications of the powdered glass formulations would be to incorporate glasses with different properties into coatings or cosmetics. To investigate the feasibility of these applications, the silver-glass powder was incorporated into polycaprolactone polymer to determine the antibacterial properties of the imbedded glass powder. Based on this investigation, the silver glass formulations reported herein have potential applications in the medical device, dental, pharmaceutical and cosmetic industries.
\end{abstract}

\section{Keywords}

Glass, Antibacterial, Silver, Borate 


\section{Introduction}

One of the major concerns in medicine is the increasing number of antibiotic resistant microorganisms [1]. A frequent source of infection by antibiotic resistant microorganisms is the hospital [2] [3]. Such hospital-acquired infections (HAIs) often arise after invasive procedures and insertion of in-dwelling medical devices or simply from contact with contaminated hospital surfaces. More frequently, these HAIs are caused by multi-drug resistant bacteria [4]. Therefore, it is desirable to prevent infections by preventing bacteria from establishing an infection in the first place. In addition, we are seeing increased prevalence of community acquired infections with multi-drug resistant bacteria, such as multi-drug resistant Staphylococcus aureus (MRSA). One approach to prevent the spread of multi-drug resistant organisms is to treat or assemble medical and household devices with antimicrobial agents that prevent the colonization of microorganisms and minimize the potential for infection [5] [6]. Silver is a powerful, broad-spectrum antimicrobial agent that has been used extensively as a topical treatment for burns [7], as an ingredient in dental amalgam [8] and in household devices [9]. Despite concern for the potential toxicity of silver ions in the human body, there is strong interest in the use of silver as a coating for in-dwelling medical devices as the benefits from using silver as an antimicrobial agent outweigh potential risk [10]. Several studies have shown that treating catheters and prosthetic devices with silver is an effective means of reducing inflammation and infection with minimal toxicity [6] [11] [12]. Typical treatment of medical devices with silver involves coating the surface of the device with silver solutions. An alternative approach is to incorporate silver containing glass particles directly into the device during manufacture. A bioactive glass has the advantage of controlling the release rate of silver and minimizing the exposure of the patient to free silver ions. Silver-releasing glasses could also be incorporated into wound dressings, sutures and other topical medical treatments. The biocompatibility of a silver containing bioglass would also make it an ideal antimicrobial ingredient in cosmetics and other skin treatments.

An exciting new development is the use of bioactive, borate-based glass in soft tissue repair [13] [14] [15] which has led to approval by the United States Food and Drug Administration (FDA) for use of one such product, Mirragen (ETS Wound Care, Rolla, MO), in treatment of acute and chronic wounds. Recent work in our laboratory has demonstrated the broad-spectrum antibacterial properties of borate-based glass doped with a variety of metals [16]. The following project complements our previous work by investigating the incorporation silver into glasses of different composition to alter the dissolution rate of the glass to control the release of silver ions and to investigate their properties in different environments and when incorporated into a polymer matrix.

\section{Materials and Methods}

Bacterial Strains. Three bacterial species commonly used as standards for an- 
timicrobial sensitivity testing were selected for this study. Escherichia coli (ATCC 25922), Pseudomonas aeruginosa (ATCC27853) and Staphylococcus aureus (ATCC 12228) were purchased from Difco Laboratories (Detroit, MI). All strains were maintained on trypticase Soy Agar and grown in trypticase soy broth (Difco Laboratories (Detroit, MI).

Zone of inhibition measurements. 100 microliters of an overnight culture of each individual organism grown in trypticase soy broth (approximately $10^{8} \mathrm{cfu} / \mathrm{ml}$ ) were spread on the surface of trypticase soy agar. The inoculated petri dishes were incubated at $37^{\circ} \mathrm{C}$ for 24 hours and effectiveness was determined by measuring the radius of inhibition surrounding the glass disk as measured from the edge of the glass disk to the edge of the region of bacterial growth.

Glass composition. Formulations of glass with and without silver were developed for medical applications with varying dissolution rates (see Table 1 for glass compositions). The formulations are informally categorized as fast (minutes), intermediate (hours) and slow releasing (days) glass. Each glass was prepared as an intact melt, ground and sifted to obtain particle sized in the range of 45 to 75 microns.

Application of glass particles to agar surfaces. In order to apply the glass to the surface of agar plates in a consistent size and quantity, $50 \mathrm{mg}$ of ground glass (particle size of $<45$ micrometer) was suspended in $1 \mathrm{~mL}$ water, phosphate-buffered saline or fetal calf serum and 200 microliters ( $10 \mathrm{mg}$ glass) was collected in a 5 $\mathrm{mm}$ diameter filter (wizard spin column, Promega, Corp. WI) by centrifugation at 15,000 $\times \mathrm{g}$ for 5 minutes. The compressed glass powder remained together as a unit and was applied to the surface of the agar plates as a disk. For time of exposure studies, glass particles remained suspended in either water, phosphate buffered saline (PBS) or Fetal Calf Serum (FCS) and aliquots were removed at specific time points, collected on a filter and applied to the surface of the agar plate.

Incorporation of silver glass in polycaprolactone (PCL) polymer. Silver glass (B-5 with $1 \% \mathrm{AgNO}_{3}$ ) and silver nitrate was added to liquid PCL solution and allowed to polymerize as a thin film. Small disks were cut out of the polymerized film using a paper punch producing $5 \mathrm{~mm}$ disks for application to the agar surface. Because of the time it takes for the PCL to polymerize, the glass particles

Table 1. Composition of borate-based bioactive glasses tested.

\begin{tabular}{ccccccc}
\hline \multirow{2}{*}{ Compound } & \multicolumn{7}{c}{ Percent Weight } \\
\cline { 2 - 7 } & B5 & B5 Ag & B8 & B8 Ag & B13 & B13 Ag \\
\hline $\mathrm{B}_{2} \mathrm{O}_{3}$ & $89 \%$ & $88 \%$ & $44.5 \%$ & $43.5 \%$ & $8 \%$ & $83 \%$ \\
$\mathrm{Na}_{2} \mathrm{O}$ & - & - & $5 \%$ & $5 \%$ & - & - \\
$\mathrm{Li}_{2} \mathrm{O}$ & $11 \%$ & $11 \%$ & $6.5 \%$ & $6.5 \%$ & $11 \%$ & $11 \%$ \\
$\mathrm{Al}_{2} \mathrm{O}_{3}$ & - & - & - & - & $5 \%$ & $5 \%$ \\
$\mathrm{SiO}_{2}$ & - & - & $44 \%$ & $44 \%$ & - & - \\
$\mathrm{Ag}_{2} \mathrm{O}$ & - & $1 \%$ & - & $1 \%$ & - & $1 \%$ \\
\hline
\end{tabular}


settled toward the bottom of the film producing a rough textured surface where the particles settled and a smooth textured surface on the top of the film. Exposure to both surfaces was tested.

\section{Results}

Silver-doped, borate-based bioactive glasses kill diverse bacteria. The use of borate-based bioactive glasses as an alternative to silicate-based bioactive glasses are of interest in medical applications due to their increased degradation rate and applications in soft tissue repair [17] [18]. Since borate bioactive glasses have a less well-established history than silicate bioactive glasses, there is still much to learn about their benefits and mechanisms in biological applications [19]. One benefit of borate bioactive glasses is that their reaction rate can be easily altered by the boron content of the glass [2]. Easily changing the reaction rate of the glass is beneficial, as this allows for the surface-reactivity of the glass to be tailored to its specific use on a case-by-case basis. For this study we have selected three silver-releasing glass formulations containing $1 \%$ silver oxide, by weight (Table 1) based on their relative silver release rate. Glass B-5 dissolves and releases silver at the highest rate. Glass B-13 dissolves and releases silver at an intermediate rate and glass B- 8 dissolves and releases silver at the slowest rate (Razvan Stefan, unpublished).

Each silver/glass formulation was assayed for effectiveness at inhibiting the growth of Escherichia coli, Staphylococcus epidermidis and Pseudomonas aeruginosa. Using standard zone of inhibition assays on trypticase soy agar plates the relative effectiveness of each formulation at inhibiting the growth of each of the three test organisms was determined. Radius of inhibition test data showed that the B-5 (fast) and B-13 (intermediate) glasses are very effective inhibitors of bacterial growth (Figure 1). The radius of inhibition was largest for the faster dissolving glasses (B-5 and B-13) but the radius of inhibition for the fastest dissolving glass (B-5) was not significantly larger than the intermediate dissolving glass (B-13). We attribute this to the mobility of the silver ions relative to the rate of growth of the bacteria resulting in a maximum size zone of inhibition. The B-8 glass dissolved slowest and produced zones of inhibition of bacterial growth much smaller than the other glasses. The small zone of inhibition produced by the B-8 glass compared to the other glass formulations is most likely due to the bacteria growing to saturation before the released silver reached a high enough concentration to kill the bacteria.

Borate-based bioactive glass alone has significant antibacterial properties. To determine the contribution of silver to the zone of inhibition, we compared the zones of inhibition for the control glass, without silver (Figure 2). For each glass formulation, the control glass demonstrated inhibition of bacterial growth but the zones were smaller than the zones created by the silver-doped glass.

Silver-doped borate bioactive glass loses effectiveness over time when suspended in deionized water. B-13 (intermediate) was the only glass that produces a measurable zone of inhibition and dissolved slowly enough to be 


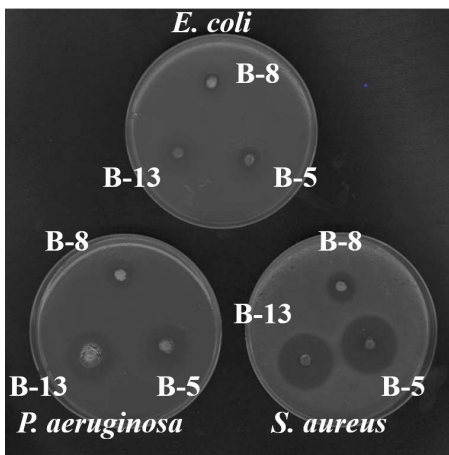

(a)

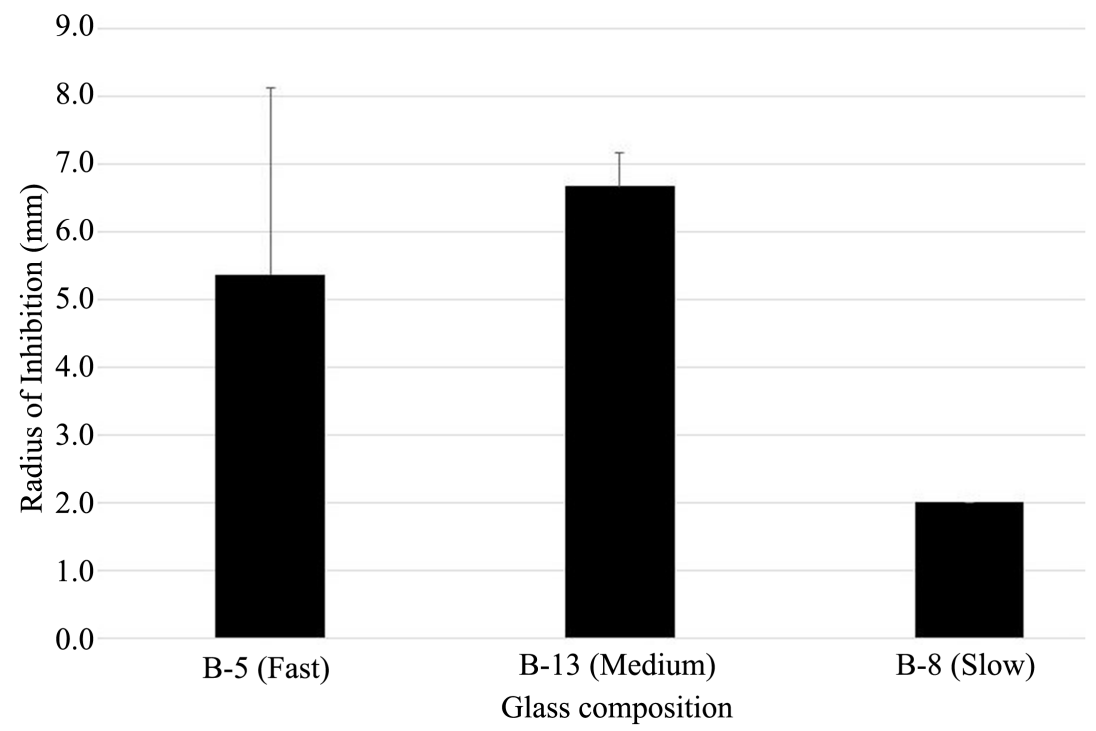

(b)

Figure 1. Silver containing glasses inhibit growth of Escherichia coli, Pseudomonas aeruginosa and Staphylococcus aureus. (a) Representative agar plates demonstrating zone of inhibition assays with each organism and each glass composition; (b) Measurement of the radius of the zone of inhibition for each glass against $E$. coli. Each bar represents a minimum of 3 independent assays.

tested at long time intervals. B-13 suspended in water for several hours remained effective at killing bacteria using our assay system. However, the size of the zone of inhibition decreases over time as the glass particles lose mass and therefore the amount of silver available to be released (Figure 3).

Phosphate-buffered saline (PBS) slows glass dissolution. Investigations of silver release in suspension showed that phosphate-buffered saline (PBS) slowed the release of silver and the rate at which the glass dissolved and lost mass (data not shown). Therefore, we tested using PBS for suspending the B-13 glass powder. PBS slowed the release of silver from the glass samples, resulting in slightly smaller zones of inhibition. However, the glass retained the ability to inhibit bacterial growth after longer periods of time in suspension (Figure 4(a)). To further simulate interaction with complex bodily fluids we suspended B-13 glass powder in fetal calf serum (FCS) and measured the impact on the size of 


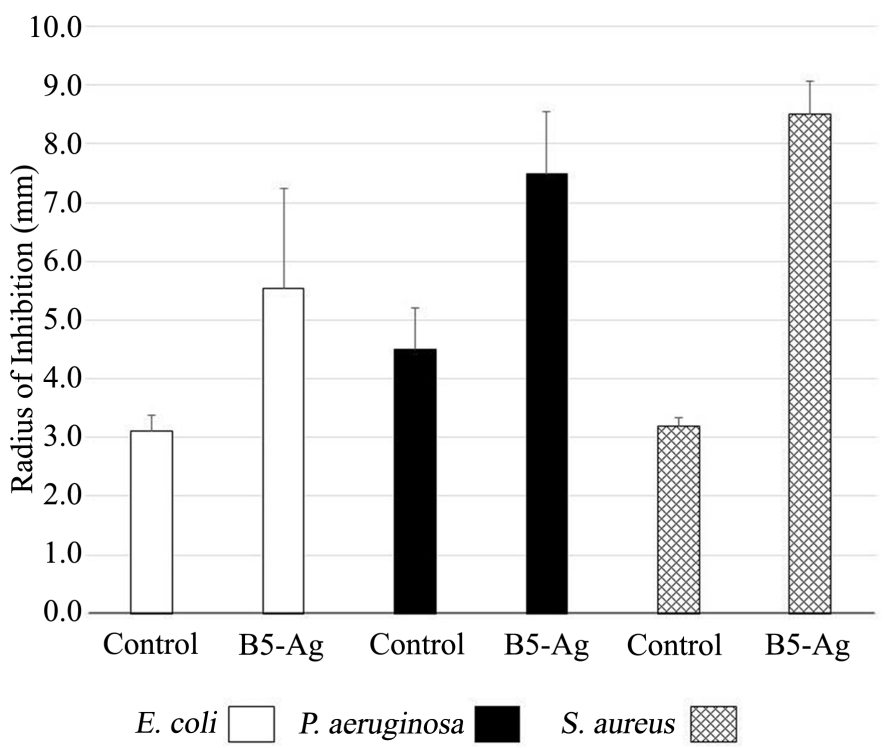

Figure 2. Comparison of B-5 control glass (no silver) versus B-5 doped with silver. Each bar represents a minimum of 3 independent assays.

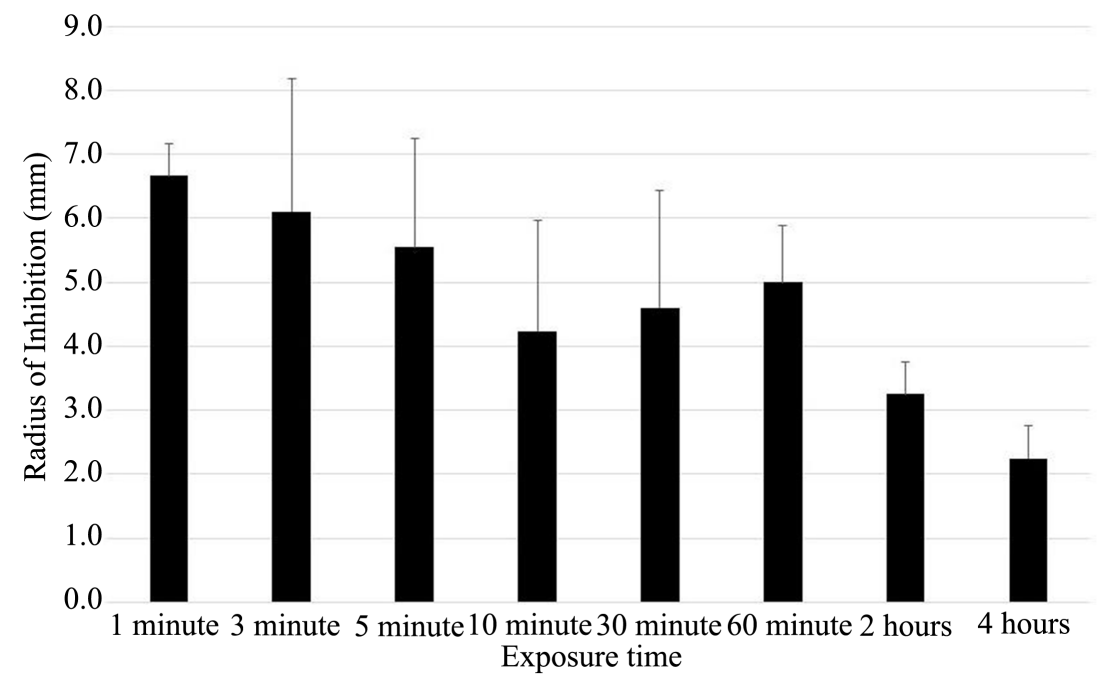

Figure 3. Silver-doped borate bioactive glass loses effectiveness over time in water. Four-hour time-course comparing the effectiveness of B-13 silver-glass over time when suspended in water. Data for $E$. coli is shown. Similar results were observed for P. aeruginosa and $S$. aureus. Each bar represents a minimum of 3 independent assays.

the zone of inhibition over time. As with suspension in PBS, suspension in FCS resulted in a reduction in the size of the zone of inhibition but the glass retained the ability to inhibit bacterial growth after longer periods of time in suspension (Figure 4(b)).

Silver-doped bioactive glass incorporated into polycaprolactone (PCL) inhibits bacterial growth. One potential application of antimicrobial silver-glass is to incorporate the silver-glass powder into other materials. Therefore, we tested the ability of a PCL polymer laced with silver-glass to inhibit the growth of our three test organisms (Figure 5). For these studies, the faster 


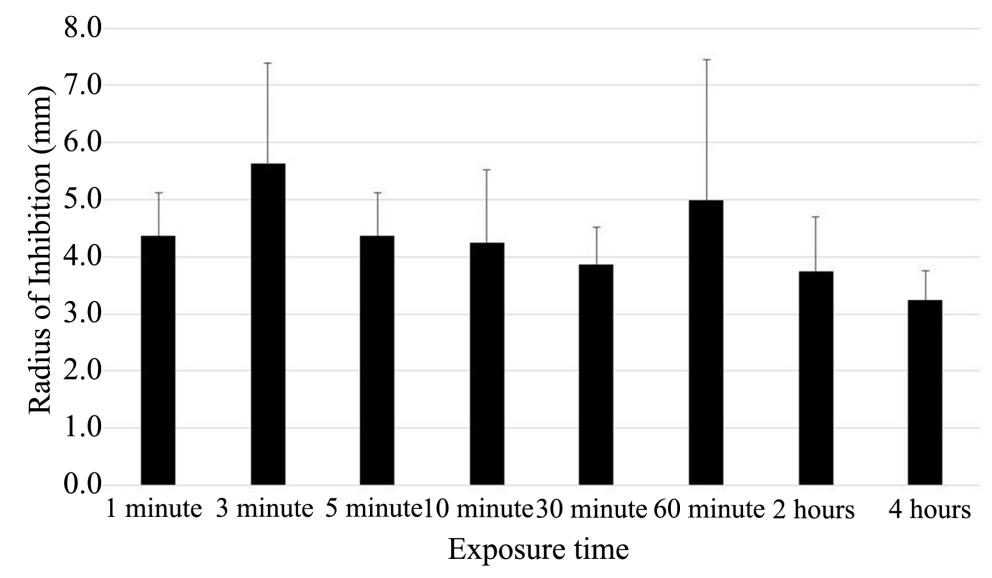

(a)

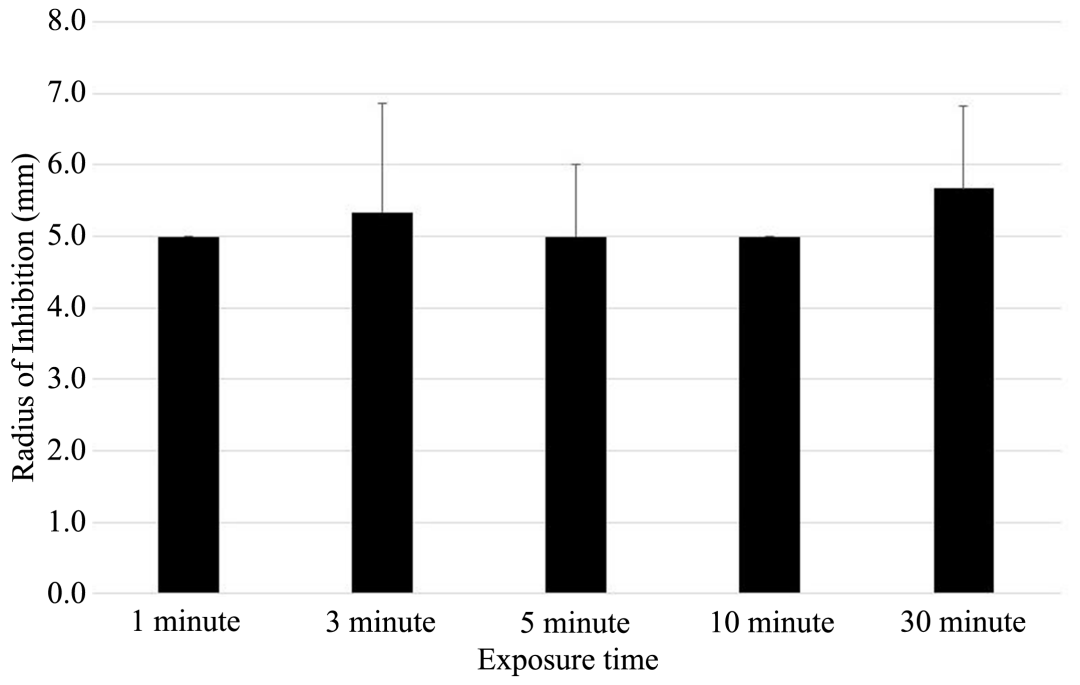

(b)

Figure 4. In phosphate-buffered saline and fetal calf serum, silver-doped bioactive glass retains effectiveness over time. Four-hour time-course comparing the effectiveness of B-13 silver-glass over time when suspended in phosphate buffered saline (PBS) (a) or fetal calf serum (FCS) (b). Data for $E$. coli is shown. Similar results were observed for $P$. aeruginosa and $S$. aureus. Each bar represents a minimum of 3 independent assays.

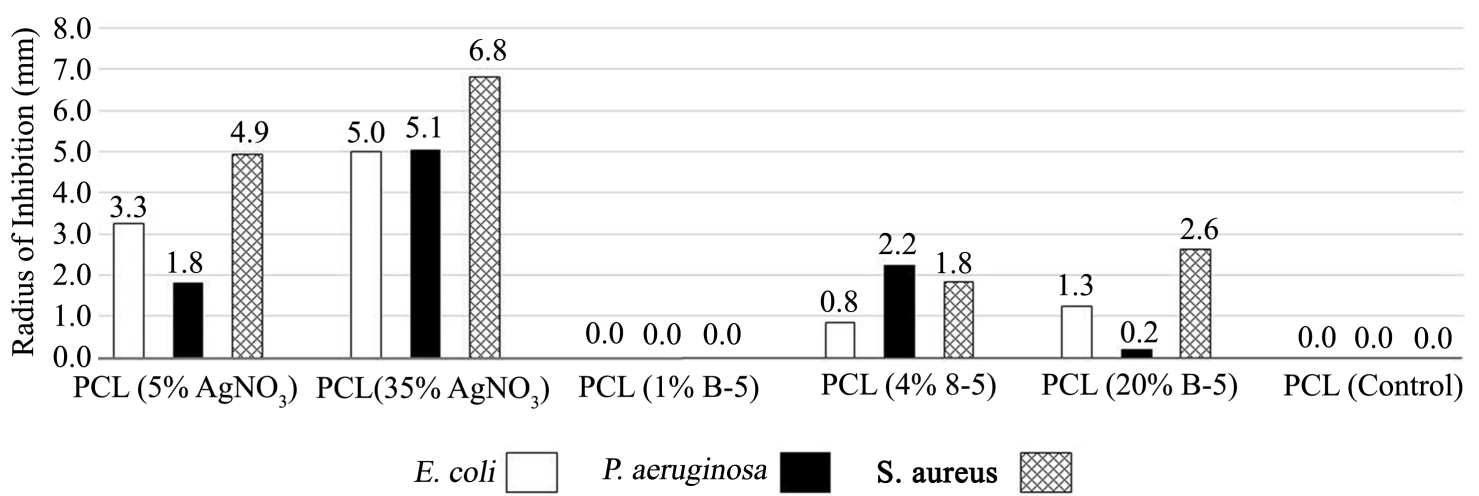

Figure 5. Silver-doped bioactive glass incorporated into polycaprolactone (PCL) inhibits bacterial growth. Polycaprolactone (PCL) as support medium for silver-glass and silver alone. Each bar represents a minimum of 3 independent assays. 
dissolving B-5 glass was used to ensure sufficient release of silver ions during the course of the experiment. Inhibition of growth for all three test-organisms was demonstrated using PCL containing silver containing glass as well as polymer containing silver alone whereas polymer containing non-silver containing glass produced no zone of inhibition. Silver ion alone was effective at concentrations as low as $5 \%$ whereas for silver-doped B-5 it was as low as $4 \%$. While the PCL containing $4 \%$ silver-doped B-5 produced a smaller zone of inhibition, the amount of silver present in the polymer containing $4 \%$ silver-doped B-5 (1\% $\mathrm{AgNO}_{3}$ ) would only contain approximately $0.04 \%$ silver. Therefore, PCL polymer was shown to be an effective delivery agent for silver alone and for silver-doped glass as an antimicrobial agent.

\section{Discussions}

The radius of inhibition assay used in this study is a simple, yet effective means of comparing the relative antimicrobial activities of various silver/glass formulations. As shown in Figure 1, all three glass formulations effectively release silver ions resulting in clear zones of inhibition. The silver-doped glass is clearly most effective against the Gram-positive $S$. aureus and less effective against the Gram-negative E. coli and $P$. aeruginosa. Although the difference in zone of inhibition between the intermediate-dissolving B-13 and the fast-dissolving B-5 was not significant, that can be attributed to the limitations of the assay. The size of the zone of inhibition is dependent upon diffusion rate of the silver through the medium, growth rate of the bacteria and the initial concentration of bacteria. Therefore, we are only measuring a fixed point in time in which the silver has diffused to a sufficient concentration to kill the bacteria before the bacteria have reached a sufficient density to be visualized as growth on the surface of the agar plate. This can be seen in the greater variability in the zone of inhibition size for B-5 glass. We interpret this to be a result of the silver being released so fast that it quickly diffuses to a concentration that is less effective at inhibiting bacterial growth. In addition, the B-5 glass dissolves so quickly that the total mass collected with the filter is somewhat variable depending on how quickly the glass suspension is filtered. This can also be observed as the compressed disc of glass becomes unstable. This variability in obtaining consistent zones of inhibition with the B-5 glass led us to use B-13 glass for investigating the various environmental parameters affecting the behavior of the silver-doped glasses and for comparing the continued effectiveness of silver-doped glass after exposure to various solutions.

It is also well-known that borate itself is an effective antimicrobial agent and is used as a preservative in various liquids used as topical agents, e.g. eye drops [20]. Figure 2 shows that the borate glass itself (B-5) is an effective antimicrobial agent, producing a zone of inhibition with all three organisms. However, the addition of silver ions to the B-5 glass extends the size of the zone of inhibition for all three organisms. This is consistent with our previous studies which showed similar results and the addition of metal ions enhanced the broad-spectrum anti- 
bacterial properties of the glass.

When used in medical and personal use products, glass will be interacting with body fluids. Initial studies of the glass formulations used in the study showed that each glass dissolved more slowly and released silver over an extended period of time when exposed to phosphate containing solutions (Razvan Stefan, unpublished). Therefore, we tested the behavior of the glass formulations in phosphate buffered saline (PBS) as well as fetal calf serum (FCS) (to simulate interaction with bodily fluids). As seen in Figure 4, suspending the glass powder in either PBS or FCS results in slightly smaller zones of inhibition but when measured after remaining in suspension for several hours the zone of inhibition did not decline as quickly as when suspended in water (Figure 3). When using bioactive glass in medical or consumer devices it is anticipated that the antimicrobial material will be released continuously and diffuse away from the device location. This means that it would be desirable to have a dynamic system in which the silver or other antimicrobial metal is released slowly over an extended period of time. These results demonstrate that it would be possible to achieve this desired effect by using a combination of glasses dissolving at different rates when they interact with bodily fluids. For example, in the case of a medical device, biofilm formation can be inhibited by the localized, controlled release of antimicrobial compounds on the surface of the device. Bioactive glass provides the ideal delivery system for such localized, controlled release. A similar effect can be achieved by incorporating the glass powder into polymer material. Figure 5 shows that it is possible to imbed silver-doped borate bioactive glass into a polymer such as polycaprolactone and achieve antibacterial properties. Demonstrating that it would be possible to create polymers imbedded with multiple glass formulations, each dissolving at a different rate, creating a material with long-lasting antimicrobial properties.

Our research demonstrates the potential use of bioactive silver doped borate glasses as effective antimicrobial additives for medical as well as general consumer products. Previous work from our laboratory demonstrated that one specific borate bioactive glass formulation doped with various silver, iodine, or gallium had broad-spectrum antibacterial properties [16]. The attractiveness of using borate based bioactive glass is the greater dissolution rate relative to silicate-based glass formulations. In addition, this dissolution rate can be readily manipulated by varying the concentration of various ions in the glass melt. The B-3 glass used in our previous work has been of great interest for its applications in soft tissue repair and regeneration [15]. Another attractive feature of the metal ion doped borate bioactive glass is that, as the bioactive glass dissolves, silver (and or other ions) is released to the surrounding medium where it inhibits the growth of diverse bacteria.

Taking advantage of the ability to manipulate the behavior of borate bioactive glass, it is possible to develop new formulations for the use of metal ion doped glass for a variety of applications. In medical devices, it can be attractive to control the release rate of silver (or other metal ions) and minimize exposure of the 
patient to free metal ions. Slow dissolving glasses could be incorporated into indwelling medical devices, wound dressings, sutures and other topical medical treatments which might benefit from the slow release of metal ions. Non-medical applications would benefit from a combination of glasses that dissolve at different rates to extend the antimicrobial properties of the material. For example the biocompatibility of bioactive borate glasses and silver would make an ideal antimicrobial ingredient in cosmetics and other skin treatments. Incorporating a combination of fast, intermediate, and slow dissolving glasses into these treatments could allow for rapid release of the antimicrobial metal on initial contact as the fast-dissolving glass reacts first. The intermediate and slow dissolving glasses would continue to release antimicrobial metals over a longer period of time, extending the usefulness of the treatment.

\section{Acknowledgements}

This research has been supported by the Missouri S \& T Graduate Center for Materials Research and the Missouri S\&T Opportunities for Undergraduate Research Experience (OURE) program.

\section{Conflicts of Interest}

The authors declare no conflicts of interest regarding the publication of this paper.

\section{References}

[1] Frieden, T. (2013) Antibiotic Resistance Threats in the United States 2013. Diane Publishing Company, Collingdale, PA.

[2] Liu, X., Xie, Z., Zhang, C., Pan, H., Rahaman, M.N., Zhang, X., Fu, Q. and Huang, W. (2010) Bioactive Borate Glass Scaffolds: In Vitro and in Vivo Evaluation for Use as a Drug Delivery System in the Treatment of Bone Infection. Journal of Materials Science: Materials in Medicine, 21, 575-582. https://doi.org/10.1007/s10856-009-3897-8

[3] Burke, J.P. (2003) Infection Control-A Problem for Patient Safety. New England Journal of Medicine, 348, 651-656. https://doi.org/10.1056/NEJMhpr020557

[4] Morris, S. and Cerceo, E. (2020) Trends, Epidemiology, and Management of Multi-Drug Resistant Gram-Negative Bacterial Infections in the Hospitalized Setting. Antibiotics, 9, Article No. 196. https://doi.org/10.3390/antibiotics9040196

[5] Singha, P., Locklin, J. and Handa, H. (2017) A Review of the Recent Advances in Antimicrobial Coatings for Urinary Catheters. Acta Biomaterialia, 50, 20-40. https://doi.org/10.1016/j.actbio.2016.11.070

[6] Lederer, J.W., Jarvis, W.R., Thomas, L. and Ritter, J. (2014) Multicenter Cohort Study to Assess the Impact of a Silver-Alloy and Hydrogel-Coated Urinary Catheter on Symptomatic Catheter-Associated Urinary Tract Infections. Journal of Wound, Ostomy \& Continence Nursing, 41, 473-480. https://doi.org/10.1097/WON.0000000000000056

[7] Klasen, H.J. (2000) A Historical Review of the Use of Silver in the Treatment of Burns. II. Renewed Interest for Silver. Burns, 26, 131-138. https://doi.org/10.1016/S0305-4179(99)00116-3 
[8] Bharti, R., Wadhwani, K., Tikku, A. and Chandra, A. (2010) Dental Amalgam: An Update. Journal of Conservative Dentistry, 13, 204-208. https://doi.org/10.4103/0972-0707.73380

[9] Sim, W., Barnard, R., Blaskovich, M.A.T. and Ziora, Z. (2018) Antimicrobial Silver in Medicinal and Consumer Applications: A Patent Review of the Past Decade (2007-2017). Antibiotics, 7, Article No. 93. https://doi.org/10.3390/antibiotics7040093

[10] Seltenrich, N. (2013) Nanosilver: Weighing the Risks and Benefits. Environmental Health Perspectives, 121, A220-A225. https://doi.org/10.1289/ehp.121-a220

[11] Kathuria, P., Moore, H.L., Prowant, B.F., Khanna, R. and Twardowski, Z. (1995) Preliminary Evaluation of Silver-Coated Peritoneal Catheters in Rats. Advances in Peritoneal Dialysis, 11, 189-192.

[12] Jansen, B., Rinck, M., Wolbring, P., Strohmeier, A. and Jahns, T. (1994) In Vitro Evaluation of the Antimicrobial Efficacy and Biocompatibility of a Silver-Coated Central Venous Catheter. Journal of Biomaterials Applications, 9, 55-70. https://doi.org/10.1177/088532829400900103

[13] Miguez-Pacheco, V., Greenspan, D., Hench, L.L. and Boccaccini, A.R. (2015) Bioactive Glasses in Soft Tissue Repair. American Ceramic Society Bulleting, 94, 27-31.

[14] Wray, P. (2011) Cotton Candy that Heals? Borate Glass Nanofibers Look Promising. American Ceramic Society Bulletin, 90, 25-29.

[15] Jung, S. (2013) Treatment of Chronic Wounds With Bioactive Borate Glass Fibers: An Introduction to Bioceramics. Imperial College Press, London.

https://doi.org/10.1142/9781908977168 0033

[16] Ottomeyer, M., Mohammadkah, A., Day, D. and Westenberg, D. (2016) BroadSpectrum Antibacterial Characteristics of Four Novel Borate-Based Bioactive Glasses. Advances in Microbiology, 6, 776-787. https://doi.org/10.4236/aim.2016.610076

[17] Rahaman, M.N., Liang, W., Day, D.E., Marion, N.W., Reilly, G.C. and Mao, J.J. (2005) Preparation and Bioactive Characteristics of Porous Borate Glass Substrates. In: Mizuno, M., Eds., Advances in Bioceramics and Biocomposites. Ceramic Engineering and Science Proceedings, 3-10. https://doi.org/10.1002/9780470291269.ch1

[18] Yao, A., Wang, D., Huang, W., Fu, Q., Rahaman, M.N. and Day, D.E. (2007) In Vitro Bioactive Characteristics of Borate-Based Glasses with Controllable Degradation Behavior. Journal of the American Ceramic Society, 90, 303-306. https://doi.org/10.1111/j.1551-2916.2006.01358.x

[19] Brown, R.F., White, J.E., McMenamin, K. and Day, D.E. (2003) Transformation of Borate Glasses into Biologically Useful Materials. Glass Technology, 44, 75-81.

[20] Houlsby, R.D., Ghajar, M. and Chavez, G.O. (1986) Antimicrobial Activity of Borate-Buffered Solutions. Antimicrobial Agents and Chemotherapy, 29, 803-806. 